\title{
Impact of COVID-19 as a vertical infection in late pregnancy
}

Hong Kong Med J 2020;26:271-2

https://doi.org/10.12809/hkmj208655

To the Editor-I am most appreciative of the comprehensive review on congenital infections in Hong Kong by Leung et $\mathrm{al}^{1}$ and the refreshing reminder of the acronym TORCH (for toxoplasmosis, others [including syphilis], rubella, cytomegalovirus and herpes simplex virus). As the coronavirus disease 2019 (COVID-19) pandemic is currently spreading rapidly worldwide, may I suggest that we add it to the TORCH list of vertical infections in pregnancy and cite a few representative early reports.

Chen et al $^{2}$ outlined nine live births from nine COVID-19 confirmed mothers, all infected in the third trimester. The maternal symptoms were mild and the babies showed no serious symptoms or signs and all tested negative for severe acute respiratory syndrome coronavirus 2 (SARS-CoV-2) infection. However, as warning signals, fetal distress occurred in two cases, five babies had lymphopenia, and two babies had raised aminotransferase levels.

Zhu et $\mathrm{al}^{3}$ were less optimistic. Of 10 babies born to nine mothers with confirmed SARS-CoV-2 infection, complications included fetal distress, preterm delivery, premature rupture of membranes, and abnormalities in amniotic fluid, umbilical cord, and placenta. Neonatal symptoms include dyspnoea $(\mathrm{n}=6)$, feeding problems, vomiting, diarrhoea, gastric bleeding $(n=4)$, and neonatal respiratory distress syndrome $(n=2)$ of which one neonate born prematurely at 35 weeks died 9 days after birth of multi-organ failure. Clinical and radiological signs were strongly suggestive of COVID-19. Nine out of the 10 babies tested negative for SARS-CoV-2 infection. Vertical transmission could not yet be established.

Later, Dong et $\mathrm{al}^{4}$ found both immunoglobulin (Ig) $\mathrm{M}$ and IgG antibodies to SARS-CoV-2 in a 2-hour-old neonate from a mother with COVID-19. Because the maternal IgM molecule is too large to cross the placenta, and it would take a few days after exposure to produce the IgM, the baby must have been exposed to the virus while in the uterus. The baby was asymptomatic except for raised interleukin (IL)-6 and IL-10 levels, and elevated white blood cell count.

More recently, Baud et $\mathrm{al}^{5}$ reported a case of second trimester (19 weeks) miscarriage in a woman with COVID-19. Placental biopsies were tested positive for SARS-CoV-2 infection and both placenta and cord showed pathological changes. The fetus showed no abnormalities and tested negative for SARS-CoV-2 infection.

Within 4 months of its emergence, COVID-19 appears to be a candidate to join the list of TORCH. More studies are needed to confirm this, especially regarding the infection in the first trimester of pregnancy, and the effect of SARS-CoV-2 infection on organogenesis and congenital defects.

\section{Author contributions}

The author contributed to the concept and design of the study, acquisition and analysis of the data, drafting of the manuscript, and critical revision of the manuscript for important intellectual content. The author had full access to the data, contributed to the study, approved the final version for publication, and takes responsibility for its accuracy and integrity.

\section{Conflicts of interest}

The author has no conflicts of interest to disclose.

\section{Funding/support}

This letter received no specific grant from any funding agency in the public, commercial, or not-for-profit sectors.

John SM Leung *, FCSHK, FHKAM (Surgery)

Cardiothoracic Surgery, St Paul's Hospital, Hong Kong

*Corresponding author: leungsiumanjohn@yahoo.com.hk

\section{References}

1. Leung KK, Hon KL, Yeung A, Leung AK, Man E. Congenital infections in Hong Kong: an overview of TORCH. Hong Kong Med J 2020;26:127-38.

2. Chen H, Guo J, Wang C, et al. Clinical characteristics and intrauterine vertical transmission potential of COVID-19 infection in nine pregnant women: a retrospective review of medical records. Lancet 2020;395:809-15.

3. Zhu H, Wang L, Fang C, et al. Clinical analysis of 10 neonates born to mothers with $2019-\mathrm{nCoV}$ pneumonia. Transl Pediatr 2020;9:51-60.

4. Dong L, Tian J, He S, et al. Possible vertical transmission of SARS-CoV-2 from an infected mother to her newborn. JAMA 2020;323:1846-8.

5. Baud D, Greub G, Favre G, et al. Second-trimester miscarriage in a pregnant woman with SARS-CoV-2 infection. JAMA 2020 Apr 30. Epub ahead of print. 